Check for updates

Cite this: RSC Adv., 2018, 8, 14171

Received 24th February 2018

Accepted 9th April 2018

DOI: $10.1039 / \mathrm{c} 8 \mathrm{ra01648h}$

rsc.li/rsc-advances

\title{
One-pot, sequential four-component synthesis of novel heterocyclic [3.3.3] propellane derivatives at room temperature $\uparrow$
}

\author{
Maryam Beyrati and Alireza Hasaninejad (D)*
}

An efficient, one-pot, two-step, four-component reaction for the synthesis of propellane derivatives is described by the condensation reaction between acenaphthenequinone, malono derivatives, primary amines and $\beta$-ketoester or $\beta$-diketone derivatives in the presence of triethylamine in ethanol at room temperature. Using this procedure, all the products were obtained in good to excellent yields.

\section{Introduction}

In modern organic chemistry, the improvement of reaction efficiency, the reduction of waste, the avoidance of toxic reagents and the responsible utilization of our resources have become critical objectives. ${ }^{1,2}$ Multi-component reactions, are useful tools for the synthesis of biologically and pharmacologically important compounds because of their environmentally friendly, green characteristics and atom efficient..$^{3-6}$ Therefore, the design and application of multi-component reactions has attracted great attention from research groups working in areas such as biological, medicinal and organic chemistry.

Heterocyclic compounds occur commonly in nature and are essential to life. ${ }^{7}$ These compounds have received considerable attention in recent times due to their wide applications. Functionalized nitrogen and oxygen-containing heterocycles are of importance as they have various industrial, pharmaceutical and biological applications. ${ }^{8-20}$

Propellanes are tricyclic compounds in which the three rings are connected to a common C-C single bond. ${ }^{21}$ Nitrogen- and oxygen-containing propellanes and their analogues constitute the structural framework of many natural and non-natural products such as periglaucine $\mathrm{A}$, hasubanone, sinoacutine and merrilactone A (Fig. 1). ${ }^{22,23}$ Propellanes due to their challenging framework and broad spectra of biological and pharmacological activities, constitute an interesting class of compounds in organic chemistry. ${ }^{24}$

Recently, there have been some reports about the synthesis of propellane compounds from ninhydrin ${ }^{25-32}$ and acenaphthoquinone. ${ }^{32-34}$ In continuation of our research on the synthesis of biologically important heterocycles and green chemistry protocols, ${ }^{35-47}$ we report a simple and efficient

Department of Chemistry, Faculty of Sciences, Persian Gulf University, Bushehr, 7516913817, Iran. E-mail: a_hasaninejad@yahoo.com

$\dagger$ Electronic supplementary information (ESI) available. See DOI: $10.1039 / \mathrm{c} 8 \mathrm{ra01648h}$ method for the synthesis of novel propellane derivatives via a one-pot, sequential four-component condensation reaction between acenaphthoquinone (1), malono derivatives (2), primary amines (4) and $\beta$-ketoester or $\beta$-diketone (5) derivatives in the presence of triethylamine in ethanol at room temperature (Scheme 1).

\section{Results and discussion}

In the first step, the reaction between acenaphthoquinone (1), malononitrile (2a), ethyl acetoacetate (5a) and benzyl amine (4c), as a model reaction, was investigated in the presence of different molar ratios of $\mathrm{Et}_{3} \mathrm{~N}$ in some solvents $(5 \mathrm{~mL})$ at room temperature and the results are summarized in Table 1. For this propose to a stirred mixture of acenaphthoquinone $1(1 \mathrm{mmol})$ and malononitrile $2 \mathrm{a}(1 \mathrm{mmol})$ in EtOH $(5 \mathrm{~mL})$ at room temperature different amount of $\mathrm{Et}_{3} \mathrm{~N}$ were added to form the corresponding Knoevenagel adduct and then ethyl acetoacetate $\mathbf{5 a}(1 \mathrm{mmol})$ and benzyl amine $4 \mathbf{c}(1 \mathrm{mmol})$ were added to obtain the corresponding product 6a. As it is shown in Table 1, higher yield and shorter reaction time were obtained when the reaction was carried out in the presence of $1 \mathrm{mmol}$ of the $\mathrm{Et}_{3} \mathrm{~N}$ in ethanol at room temperature (Table 1, entry 4). It should be mention that simultaneity of this four-component condensation reaction was studied in the presence of $1 \mathrm{mmol} \mathrm{Et}_{3} \mathrm{~N}$ in $\mathrm{EtOH}$ at room temperature and we observed that benzyl amine has remained
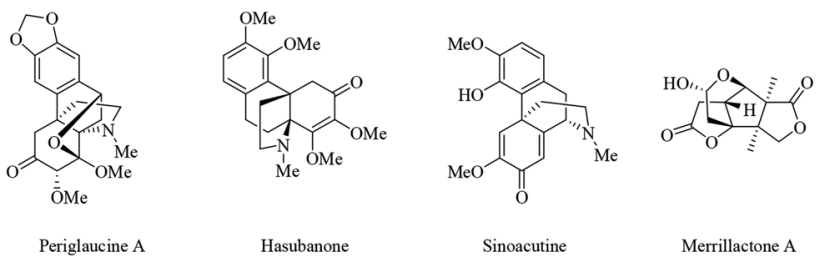

Fig. 1 Typical natural and biological products containing propellane skeleton. 


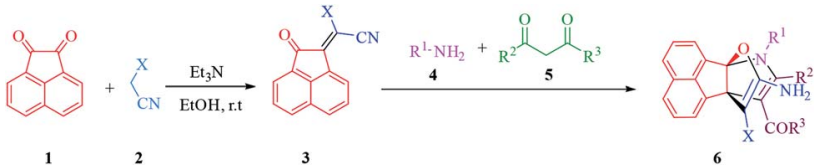

Scheme 1 The synthesis of propellane derivatives via the reaction between acenaphthoquinone, malono derivatives, dicarbonyl compounds and primary amines in the presence of $\mathrm{Et}_{3} \mathrm{~N}$ in $\mathrm{EtOH}$ at room temperature.

intact in this condition and the corresponding propellane was not formed.

In the next step, the scope and efficiency of the process was explored under the optimized conditions. For this purpose, acenaphthoquinone was condensed with alkyl malonates, aliphatic and aromatic amines and dicarbonyl compounds in the presence of $\mathrm{Et}_{3} \mathrm{~N}(1 \mathrm{mmol})$ to afford the corresponding products 6 . The structural diversity of reactants is summarized in Fig. 2 and the results are displayed in Table 2 .

The synthetic pathway for the synthesis of titled compounds is consisting of two steps. At first, compound 3 are obtained from acenaphthoquinone $\mathbf{1}$ and alkyl malonates 2 . Then, the resulting products are treated with primary amines 4 and $\beta$ dicarbonyl compounds $\mathbf{5}$ to afford the related propellane derivatives as the desired products. As Table 2 indicates, a variety of alkyl malonates, aliphatic and aromatic amines and $\beta$-dicarbonyl compounds were successfully applied in this process to afford the corresponding propellane derivatives as novel compounds in good to excellent yields.

To demonstrate the generality and scope of this method, malono derivatives, various acyclic $\beta$-dicarbonyl compounds such as methyl acetoacetate, ethyl acetoacetate and ethyl benzoylacetate were treated with a broad range of aliphatic and aromatic amines in the presence of $\mathrm{Et}_{3} \mathrm{~N}(1 \mathrm{mmol})$ in $\mathrm{EtOH}$ at room temperature and the results are shown in Table 2 . Use of malononitrile leads to shorter reaction times and higher yields compared to application of other alkylmalonates such as methyl

Table 1 Optimization of the reaction conditions ${ }^{a}$

\begin{tabular}{lllll}
\hline & $\begin{array}{l}\text { Amount of } \mathrm{Et}_{3} \mathrm{~N} \\
(\mathrm{mmol})\end{array}$ & Solvent & Time (h) & Yield $^{b}(\%)$ \\
\hline 1 & - & EtOH & 5 & - \\
2 & 0.1 & $\mathrm{EtOH}$ & 5 & 45 \\
3 & 0.5 & $\mathrm{EtOH}$ & 5 & 87 \\
4 & 1.0 & $\mathrm{EtOH}$ & 1.2 & 91 \\
5 & 1.5 & $\mathrm{EtOH}$ & 1.2 & 91 \\
6 & 1.0 & $\mathrm{H}_{2} \mathrm{O}$ & 2.0 & 70 \\
7 & 1.0 & $\mathrm{DMF}^{2}$ & 4.0 & 60 \\
8 & 1.0 & $\mathrm{MeOH}^{2}$ & 1.5 & 85 \\
9 & 1.0 & $\mathrm{CHCl}_{3}$ & 4.0 & 40 \\
10 & 1.0 & $\mathrm{THF}$ & 4.0 & 55 \\
11 & 1.0 & $\mathrm{CH}_{3} \mathrm{CN}$ & 40 & 45
\end{tabular}

${ }^{a}$ Triethylamine $(1.0 \mathrm{mmol})$ was added to a stirred mixture of acenaphthoquinone 1 (1 mmol), malononitrile $2 \mathrm{a}(1 \mathrm{mmol})$ in $\mathrm{EtOH}$ $(5 \mathrm{~mL})$ at room temperature and after about $10 \mathrm{~min}$ to form the corresponding Knoevenagel adduct, then ethyl acetoacetate 5a (1 $\mathrm{mmol})$ and benzyl amine $4 \mathbf{c}(1 \mathrm{mmol})$ were added to obtain the corresponding product $\mathbf{6 a} .{ }^{b}$ Isolated yields. cyanoacetate or ethyl cyanoacetate (compare $\mathbf{6 b}$ with $\mathbf{6 p}$ and $\mathbf{6 l}$ with 6q). As it is shown in Table 2, benzyl amines bearing electron-donating or -withdrawing substituents gave the desired propellanes in high yields and in short reaction times and it seems that when the methoxy group is placed on the aromatic ring, reactivity is better than when the chlorine group is on the ring. Moreover, the presence of 3,5-dimethyl group on the aromatic ring has the best efficiency. Some aromatic amines were also screened to carry out the four-component reaction by this method and the results are listed in Table 2 (entries $\mathbf{6 h - 6 j}$ ). Generally, aliphatic amines are more reactive than aromatic amines. Ortho-substituted anilines, whatever the nature of the substituted groups, required a longer reaction period. For instance, reaction of acenaphthenequinone, malononitrile and 2,6-dimethylaniline with ethyl acetoacetate gave the desired product (6j) in 70\% yield after $3.1 \mathrm{~h}$. Moreover, aliphatic amines such as ethyl amine and butyl amine were used for the synthesis of the corresponding product in excellent yields (Table 2, entries 6l-6o). In the same way, Some cyclic and acyclic 1.3-dicarbonyl compounds were also screened to carry out the one pot fourcomponent reaction by this method and the results are listed in Table 2. As it is shown in Table 2, aliphatic $\beta$-keto esters are more reactive than aromatic $\beta$-keto esters (compare $\mathbf{6 a}$ and $\mathbf{6 c}$ ) and no products were obtained when cyclic 1.3-dicarbonyl compounds were involved in this one-pot room temperature reaction.

Acenaphthoquinone 1

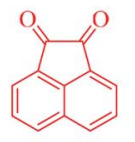

Malono derivative 2

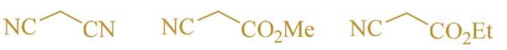

(a)

(b)

(c)

Primary amines 4

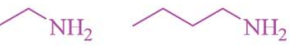

(a)

(b)

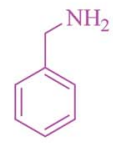

(c)

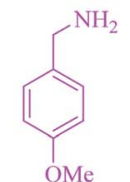

(d)

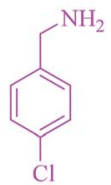

(e)

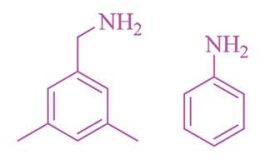

(f)

(g)

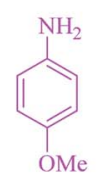

(h)

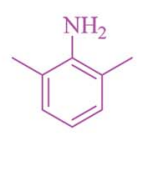

(i)

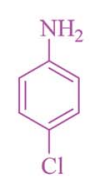

(j)
Dicarbonyl compounds 5

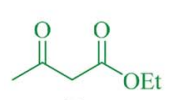

(a)

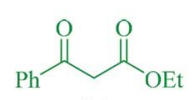

(b)

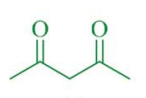

(c)
Fig. 2 Diversity elements employed for synthesis of propellane derivatives. 
Table 2 One-pot, sequential four component synthesis of propellane derivatives in the presence of $\mathrm{Et}_{3} \mathrm{~N}(1 \mathrm{mmol})$ in $\mathrm{EtOH}$ at room temperature..$^{a, b}$ 6d, $1.0 \mathrm{~h}, 90 \%$

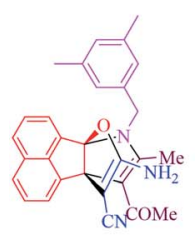

$6 g, 1.0 \mathrm{~h}, 95 \%$

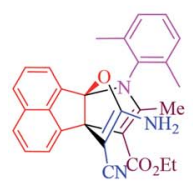

$6 \mathbf{j}, 3.1 \mathrm{~h}, 70 \%$

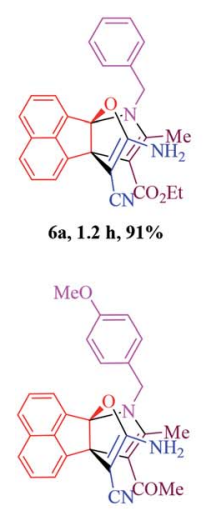

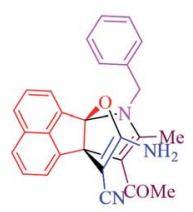

6b, $1.2 \mathrm{~h}, 92 \%$

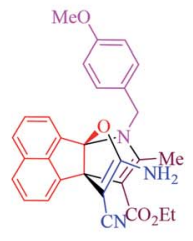

6 e, 1.2 h, $92 \%$

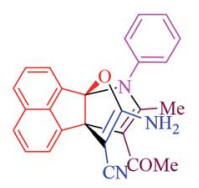

6h, 2.2 h, $79 \%$

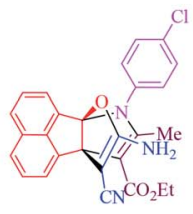

6k, 2.7 h, 77\%

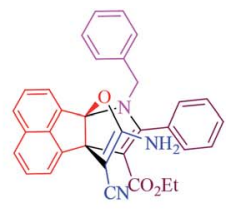

6c, 2.0 h, $87 \%$

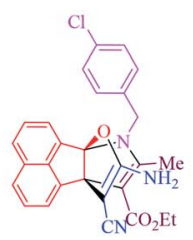

6f, $1.5 \mathrm{~h}, 86 \%$

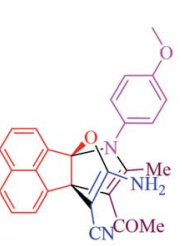

6i, 2.0 h, $83 \%$

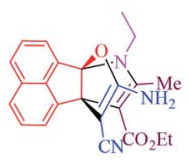

$61,1.2 \mathrm{~h}, 91 \%$

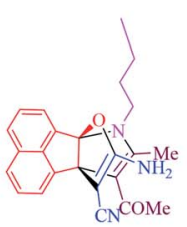

$60,1.3 \mathrm{~h}, 89 \%$ 6m, 1.2 h, $93 \%$

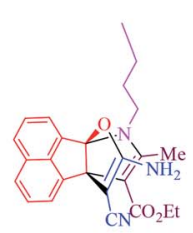

6n, $1.5 \mathrm{~h}, 87 \%$

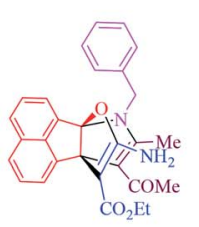

6p, $2.0 \mathrm{~h}, 86 \%$

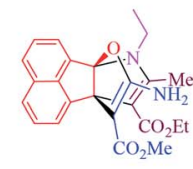

6q, 2.2 h, $85 \%$
${ }^{a}$ Triethylamine $(1.0 \mathrm{mmol})$ was added to a stirred mixture of acenaphthoquinone 1 ( $1 \mathrm{mmol})$, malono derivatives $2(1 \mathrm{mmol})$ in $\mathrm{EtOH}(5 \mathrm{~mL})$ at room temperature to form the corresponding Knoevenagel adduct (about 10-30 min), then dicarbonyl compound 5 $(1 \mathrm{mmol})$ and primary amine $4(1 \mathrm{mmol})$ were added to obtain the desired product $6 .{ }^{b}$ Isolated yields.

The proposed mechanism for the synthesis of propellane compounds 6 in the presence of $\mathrm{Et}_{3} \mathrm{~N}$ is shown in Scheme 2 . Based on this mechanism, $\mathrm{Et}_{3} \mathrm{~N}$ is effective catalyst for the formation of olefin (3) which readily prepares in situ by Knoevenagel condensation of acenaphthoquinone (1) with the active

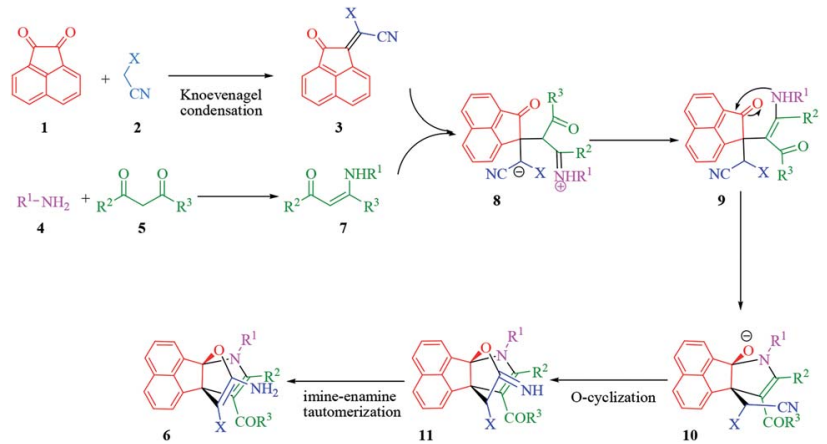

Scheme 2 The proposed mechanism for the synthesis of propellane 6 in the presence of $\mathrm{Et}_{3} \mathrm{~N}$.

methylene of compound (2). Next, the formation of enamine (7) occurs through condensation of amine (4) with dicarbonyl compound (5). Then, the enamine (7) attacks to olefin (3) in a Michael addition to produce intermediate (8). After proton transfer and tautomerization, intermediate (8) converts to intermediate (10), through intramolecular cyclization by nitrogen attack of enamine to carbonyl group. In this stage, nucleophilic addition of amino group to $\mathrm{C}=\mathrm{O}$ bond afforded intermediate (10). Then, intramolecular $O$-cyclization and the tautomerization of imino group to amino group lead to propellanes (6).

\section{Experimental}

All chemicals were purchased from Merck or Fluka chemical companies. The ${ }^{1} \mathrm{H}$ NMR (400 MHz) and ${ }^{13} \mathrm{C}$ NMR $(100 \mathrm{MHz})$ were run on a Bruker Avance 400. Melting points were recorded on a Stuart Scientific Apparatus SMP3 (UK) in open capillary tubes. Elemental $\mathrm{C}, \mathrm{H}$ and $\mathrm{N}$ analyses were performed using a Costech CHNS-O elemental analyzer.

\section{General procedure for the synthesis of propellane derivatives} 6

Triethylamine $(1.0 \mathrm{mmol})$ was added to a stirred mixture of acenaphthoquinone 1 ( $1 \mathrm{mmol})$, malono derivatives 2 ( $1 \mathrm{mmol})$ in $\mathrm{EtOH}(5 \mathrm{~mL})$ and the reaction mixture was stirred to complete the formation of related Knoevenagel adduct 3 (monitored by TLC). Subsequently, dicarbonyl compound 5 (1 mmol) and primary amine 4 ( $1 \mathrm{mmol}$ ) were added to this reaction mixture at once and dropwise over $10 \mathrm{~min}$, respectively and reacted at room temperature for the appropriate amount of time (see Table 2). After completion of the reaction confirmed by TLC, the reaction mixture was filtered and washed with ethanol to afford the pure product $\mathbf{6 a - 1}$.

Ethyl-11-amino-7-benzyl-10-cyano-8-methyl-7H-6b,9a-(epoxyetheno)acenaphtho[1,2-b]pyrrole-9-carboxylate 6a. White powder, $\mathrm{mp}=249-251{ }^{\circ} \mathrm{C},{ }^{1} \mathrm{H}$ NMR $\left(\mathrm{DMSO}-d_{6}, 400 \mathrm{MHz}\right)$ $\delta(\mathrm{ppm}): 1.45(\mathrm{t}, J=6.6 \mathrm{~Hz}, 3 \mathrm{H}), 2.25(\mathrm{~s}, 3 \mathrm{H}), 4.04(\mathrm{q}, J=7.2 \mathrm{~Hz}$, $2 \mathrm{H}), 4.54$ (d, $J=16.0 \mathrm{~Hz}, 1 \mathrm{H}), 4.60$ (d, $J=16.0 \mathrm{~Hz}, 1 \mathrm{H}), 7.57$ (s, 2H), 7.66-7.71 (m, 3H), 7.73-7.81 (m, 4H), 7.89 (d, $J=8.0 \mathrm{~Hz}$, $2 \mathrm{H}), 8.04(\mathrm{~d}, J=7.2 \mathrm{~Hz}, 2 \mathrm{H}) .{ }^{13} \mathrm{C}$ NMR (DMSO- $\left.d_{6}, 100 \mathrm{MHz}\right)$ 
$\delta(\mathrm{ppm}): 14.4,14.6,58.1,60.5,61.2,70.7,79.9,110.4,118.4$, 120.6 , 121.0, 124.7, 125.8, 127.8, 129.2, 129.6, 132.0, 135.8, 136.3, 142.7, 147.2, 147.6, 147.7, 162.4, 166.5, 166.9, 168.0, 168.1. Anal. calcd for $\mathrm{C}_{28} \mathrm{H}_{23} \mathrm{~N}_{3} \mathrm{O}_{3}$ : C, 74.82; H, 5.16; N, 9.35\%. Found: C, 74.85; H, 5.14; N, 9.33\%. IR (KBr) $\left(\nu_{\max }, \mathrm{cm}^{-1}\right): 3358$ and $3192\left(\mathrm{NH}_{2}\right), 2193(\mathrm{CN}), 1687(\mathrm{C}=\mathrm{O}), 1652(\mathrm{OC}=\mathrm{C})$.

9-Acetyl-11-amino-7-benzyl-8-methyl-7H-6b,9a-(epoxyetheno)acenaphtho[1,2-b]pyrrole-10-carbonitrile $6 \mathrm{~b}$. White powder, $\mathrm{mp}=250$ $252{ }^{\circ} \mathrm{C},{ }^{1} \mathrm{H}$ NMR (DMSO- $\left.d_{6}, 400 \mathrm{MHz}\right) \delta(\mathrm{ppm}): 2.32$ (s, 3H), 2.36 (s, $3 \mathrm{H}), 4.74(\mathrm{~d}, J=15.6 \mathrm{~Hz}, 1 \mathrm{H}), 4.99$ (d, $J=15.6 \mathrm{~Hz}, 1 \mathrm{H}), 7.45(\mathrm{~s}, 2 \mathrm{H})$, $7.63(\mathrm{t}, J=7.4 \mathrm{~Hz}, 2 \mathrm{H}), 7.69-7.71(\mathrm{~m}, 2 \mathrm{H}), 7.73-7.77(\mathrm{~m}, 3 \mathrm{H}), 7.85(\mathrm{~d}$, $J=7.6 \mathrm{~Hz}, 2 \mathrm{H}$ ), 8.02 (d, $J=8.0 \mathrm{~Hz}, 2 \mathrm{H}) .{ }^{13} \mathrm{C}$ NMR (DMSO- $d_{6}, 100$ $\mathrm{MHz}) \delta$ (ppm): 15.9, 30.3, 58.4, 59.1, 72.2, 79.8, 116.4, 116.6, 118.5, 120.4, 121.4, 121.6, 124.4, 124.9, 127.7, 128.9, 129.5, 131.9, 135.9, 136.3, 143.0, 148.0, 156.6, 157.1, 166.5, 167.2, 192.7. Anal. calcd for $\mathrm{C}_{27} \mathrm{H}_{21} \mathrm{~N}_{3} \mathrm{O}_{2}$ : C, 77.31; H, 5.05; N, 10.02\%. Found: C, 77.32; H, 5.02; N, $10.05 \%$.

Ethyl-11-amino-7-benzyl-10-cyano-8-phenyl-7H-6b,9a-(epoxyetheno)acenaphtho[1,2-b]pyrrole-9-carboxylate $6 c$. White powder, $\mathrm{mp}=247-249{ }^{\circ} \mathrm{C},{ }^{1} \mathrm{H}$ NMR $\left(\mathrm{DMSO}-d_{6}, 400 \mathrm{MHz}\right.$ ) $\delta(\mathrm{ppm}): 1.38(\mathrm{t}, J=7.0 \mathrm{~Hz}, 3 \mathrm{H}),(\mathrm{q}, J=6.8 \mathrm{~Hz}, 2 \mathrm{H}), 4.92(\mathrm{~d}, J=$ $16.0 \mathrm{~Hz}, 1 \mathrm{H}), 4.98(\mathrm{~d}, J=16.0 \mathrm{~Hz}, 1 \mathrm{H}), 7.43(\mathrm{t}, J=7.4 \mathrm{~Hz}, 3 \mathrm{H})$, $7.51(\mathrm{t}, J=7.0 \mathrm{~Hz}, 3 \mathrm{H}), 7.66(\mathrm{~s}, 2 \mathrm{H}), 7.70-7.77(\mathrm{~m}, 4 \mathrm{H}), 7.83(\mathrm{~d}, J$ $=6.8 \mathrm{~Hz}, 1 \mathrm{H}), 7.87(\mathrm{~d}, J=7.2 \mathrm{~Hz}, 1 \mathrm{H}), 7.93(\mathrm{~d}, J=8.0 \mathrm{~Hz}, 2 \mathrm{H})$, 8.07 (d, $J=8.0 \mathrm{~Hz}, 2 \mathrm{H}) .{ }^{13} \mathrm{C}$ NMR (DMSO- $\left.d_{6}, 100 \mathrm{MHz}\right) \delta(\mathrm{ppm})$ : 15.3, 43.6, 47.1, 47.2, 72.2 , 79.9, 113.0, 116.3, 117.3, 117.5, 121.2, $123.4,125.4,125.7,127.0,127.7,127.9,128.3,130.3,134.5$, $134.7,135.6,139.3,147.4,152.6,155.4,158.5,158.5,159.1$, 159.3, 162.2, 168.1. Anal. calcd for $\mathrm{C}_{33} \mathrm{H}_{25} \mathrm{~N}_{3} \mathrm{O}_{3}$ : C, 77.48; $\mathrm{H}$, 4.93; N, 8.21\%. Found: C, 77.51; H, 4.91; N, 8.19\%.

9-Acetyl-11-amino-7-(4-methoxybenzyl)-8-methyl-7H-6b,9a-(epoxyetheno)acenaphtho[1,2-b]pyrrole-10-carbonitrile $6 d$. White powder, $\mathrm{mp}=249-251{ }^{\circ} \mathrm{C},{ }^{1} \mathrm{H}$ NMR $\left(\mathrm{DMSO}-d_{6}, 400 \mathrm{MHz}\right.$ ) $\delta(\mathrm{ppm}): 2.32(\mathrm{~s}, 3 \mathrm{H}), 2.37(\mathrm{~s}, 3 \mathrm{H}), 3.75(\mathrm{~s}, 3 \mathrm{H}), 4.67(\mathrm{~d}, J=$ $16.0 \mathrm{~Hz}, 1 \mathrm{H}), 4.78$ (d, $J=16.0 \mathrm{~Hz}, 1 \mathrm{H}), 7.35$ (d, $J=6.8 \mathrm{~Hz}, 2 \mathrm{H})$, 7.55-7.59 (m, 2H), $7.77(\mathrm{t}, J=7.6 \mathrm{~Hz}, 2 \mathrm{H}), 7.84-7.89(\mathrm{~m}, 2 \mathrm{H})$, 8.09 (s, 2H), 8.17 (d, $J=7.6 \mathrm{~Hz}, 2 \mathrm{H}) .{ }^{13} \mathrm{C}$ NMR (DMSO- $d_{6}, 100$ $\mathrm{MHz}) \delta(\mathrm{ppm}): 15.9,30.3,57.6,58.0,58.4,72.2,79.9,118.5$, $120.4,121.4,121.7,124.5$, 124.9, 127.7, 128.9, 129.5, 131.9, $135.9,136.3,140.0,140.1,140.6,140.8,143.8,166.6,167.2$, 168.6, 192.7. Anal. calcd for $\mathrm{C}_{28} \mathrm{H}_{23} \mathrm{~N}_{3} \mathrm{O}_{3}: \mathrm{C}, 74.82 ; \mathrm{H}, 5.16 ; \mathrm{N}$, 9.35\%. Found: C, 74.79; H, 5.17; N, 9.38\%. IR (KBr) $\left(\nu_{\max }, \mathrm{cm}^{-1}\right)$ : 3361 and $3101\left(\mathrm{NH}_{2}\right), 2195(\mathrm{CN}), 1711(\mathrm{C}=\mathrm{O}), 1644(\mathrm{OC}=\mathrm{C})$.

Ethyl-11-amino-10-cyano-7-(4-methoxybenzyl)-8-methyl$7 \boldsymbol{H}$-6b,9a-(epoxyetheno)acenaphtho[1,2-b]pyrrole-9-

carboxylate 6e. White powder, $\mathrm{mp}=251-253{ }^{\circ} \mathrm{C},{ }^{1} \mathrm{H} \mathrm{NMR}$ (DMSO- $\left.d_{6}, 400 \mathrm{MHz}\right) \delta(\mathrm{ppm}): 0.57(\mathrm{t}, J=7.6 \mathrm{~Hz}, 3 \mathrm{H}), 1.90(\mathrm{~s}$, $3 \mathrm{H}), 3.10(\mathrm{~s}, 3 \mathrm{H}), 3.45-3.49(\mathrm{~m}, 1 \mathrm{H}), 3.79-3.82(\mathrm{~m}, 1 \mathrm{H}), 4.77$ (d, $=16.0 \mathrm{~Hz}, 1 \mathrm{H}), 5.03(\mathrm{~d}, J=15.6 \mathrm{~Hz}, 1 \mathrm{H}), 7.57(\mathrm{~s}, 2 \mathrm{H}), 7.66-7.71$ $(\mathrm{m}, 2 \mathrm{H}), 7.73-7.76(\mathrm{~m}, 2 \mathrm{H}), 7.79(\mathrm{t}, J=6.0 \mathrm{~Hz}, 2 \mathrm{H}), 7.88(\mathrm{~d}, J=$ $8.0 \mathrm{~Hz}, 2 \mathrm{H}$ ), 8.03 (d, $J=7.6 \mathrm{~Hz}, 2 \mathrm{H}) .{ }^{13} \mathrm{C}$ NMR (DMSO- $d_{6}, 100$ MHz) $\delta$ (ppm): 14.4, 14.6, 47.5, 69.4, 70.0, 73.1, 73.3, 79.7, 106.1, 116.2 , 117.6, 121.2, 125.1, 127.0, 127.7, 127.9, 128.2, 130.2, $134.7,135.5,139.3,147.4,158.7,159.3,159.8,160.7,162.3$, 165.0, 168.8. Anal. calcd for $\mathrm{C}_{29} \mathrm{H}_{25} \mathrm{~N}_{3} \mathrm{O}_{4}: \mathrm{C}, 72.64 ; \mathrm{H}, 5.25 ; \mathrm{N}$, $8.76 \%$. Found: C, 72.62; H, 5.23; N, 8.79\%.
Ethyl-11-amino-7-(4-chlorobenzyl)-10-cyano-8-methyl-7H-6b,9a(epoxyetheno)acenaphtho[1,2-b]pyrrole-9-carboxylate 6 . White powder, $\mathrm{mp}=249-251{ }^{\circ} \mathrm{C},{ }^{1} \mathrm{H}$ NMR $\left(\mathrm{DMSO}-d_{6}, 400 \mathrm{MHz}\right)$ $\delta(\mathrm{ppm}): 1.44(\mathrm{t}, J=7.0 \mathrm{~Hz}, 3 \mathrm{H}), 2.25(\mathrm{~s}, 3 \mathrm{H}), 4.11-4.17(\mathrm{~m}, 2 \mathrm{H})$, $4.42(\mathrm{~d}, J=16.0 \mathrm{~Hz}, 1 \mathrm{H}), 4.48(\mathrm{~d}, J=16.0 \mathrm{~Hz}, 1 \mathrm{H}), 7.77(\mathrm{~s}, 2 \mathrm{H})$, 7.85-7.91 (m, 2H), 7.92-7.96 (m, 3H), $7.99(\mathrm{t}, J=6.0 \mathrm{~Hz}, 1 \mathrm{H})$, $8.08(\mathrm{~d}, J=8.4 \mathrm{~Hz}, 2 \mathrm{H}), 8.23(\mathrm{~d}, J=8.0 \mathrm{~Hz}, 2 \mathrm{H}) .{ }^{13} \mathrm{C} \mathrm{NMR}$ $\left(\mathrm{DMSO}-d_{6}, 100 \mathrm{MHz}\right) \delta(\mathrm{ppm}): 14.4,14.6,58.1,60.6,60.6,70.7$, 79.9, 110.4, 118.4 120.6, 121.0, 124.7, 125.8, 127.8, 129.1, 129.6, $132.0,135.8,136.3,142.7,154.8,155.1,155.8,157.5,157.8$, 166.6, 167.2, 167.3. Anal. calcd for $\mathrm{C}_{28} \mathrm{H}_{22} \mathrm{ClN}_{3} \mathrm{O}_{3}$ : C, 69.49; $\mathrm{H}$, 4.58; Cl, 7.33; N, 8.68\%. Found: C, 69.50; H, 4.56; N, 8.70\%.

9-Acetyl-11-amino-7-(3,5-dimethylbenzyl)-8-methyl-7H-6b,9a(epoxyetheno)acenaphtho[1,2-b]pyrrole-10-carbonitrile 6g. White powder, $\mathrm{mp}=251-253{ }^{\circ} \mathrm{C},{ }^{1} \mathrm{H}$ NMR $\left(\mathrm{DMSO}-d_{6}, 400 \mathrm{MHz}\right)$ $\delta(\mathrm{ppm}): 2.05$ (s, 3H), $2.10(\mathrm{~s}, 3 \mathrm{H}), 2.31(\mathrm{~s}, 3 \mathrm{H}), 2.36(\mathrm{~s}, 3 \mathrm{H}), 4.96$ $(\mathrm{d}, J=16.0 \mathrm{~Hz}, 1 \mathrm{H}), 5.02(\mathrm{~d}, J=16.0 \mathrm{~Hz}, 1 \mathrm{H}), 6.83(\mathrm{~d}, J=7.6 \mathrm{~Hz}$, $1 \mathrm{H}), 7.02(\mathrm{t}, J=7.4 \mathrm{~Hz}, 1 \mathrm{H}), 7.21-7.35(\mathrm{~m}, 2 \mathrm{H}), 7.49(\mathrm{~d}, J=$ $7.2 \mathrm{~Hz}, 2 \mathrm{H}), 7.58(\mathrm{~d}, J=9.2 \mathrm{~Hz}, 1 \mathrm{H}), 7.79(\mathrm{~s}, 2 \mathrm{H}), 7.85(\mathrm{dd}, J=$ $2.4 \mathrm{~Hz}, 8.8 \mathrm{~Hz}, 1 \mathrm{H}), 8.02$ (d, $J=2.4 \mathrm{~Hz}, 1 \mathrm{H}) .{ }^{13} \mathrm{C}$ NMR (DMSO- $d_{6}$, $100 \mathrm{MHz}) \delta$ (ppm): 14.1, 20.2, 20.5, 30.3, 47.4, 59.3, 71.9, 79.7, $103.9,108.4,112.9,116.9,122.4$, 123.4, 123.6, 125.4, 127.7, $128.4,128.7,134.1,134.5,137.2$, 145.4, 152.4, 154.5, 158.4, 159.2, 167.5, 198.2. Anal. calcd for $\mathrm{C}_{29} \mathrm{H}_{25} \mathrm{~N}_{3} \mathrm{O}_{2}$ : C, 77.83; H, 5.63; N, 9.39\%. Found: C, 77.80; H, 5.65; N, 9.41\%.

9-Acetyl-11-amino-8-methyl-7-phenyl-7H-6b,9a-(epoxyetheno) acenaphtho[1,2-b]pyrrole-10-carbonitrile $6 \mathbf{h}$. White powder, $\mathrm{mp}$ $=247-249{ }^{\circ} \mathrm{C},{ }^{1} \mathrm{H}$ NMR (DMSO- $\left.d_{6}, 400 \mathrm{MHz}\right) \delta(\mathrm{ppm}): 2.31(\mathrm{~s}$, $3 \mathrm{H}), 2.36(\mathrm{~s}, 3 \mathrm{H}), 7.51-7.60(\mathrm{~m}, 3 \mathrm{H}), 7.78-7.82(\mathrm{~m}, 2 \mathrm{H}), 7.88(\mathrm{~s}$, $2 \mathrm{H}), 7.97(\mathrm{dd}, J=1.4 \mathrm{~Hz}, 7.8 \mathrm{~Hz}, 2 \mathrm{H}), 8.23(\mathrm{dd}, J=2.2 \mathrm{~Hz}, 8.6 \mathrm{~Hz}$, $2 \mathrm{H}), 8.38$ (d, $J=2.4 \mathrm{~Hz}, 2 \mathrm{H}) .{ }^{13} \mathrm{C}$ NMR (DMSO- $d_{6}, 100 \mathrm{MHz}$ ) $\delta$ (ppm): 15.8, 30.2, 57.2, 71.4, 79.8, 101.7, 109.6, 112.9, 117.2, 117.5 , 123.2, 123.4, 124.6, 125.5, 127.6, 127.8, 128.9, 129.5, 132.8, 134.3, 136.4, 143.2, 152.6, 155.8, 158.9, 199.0. Anal. calcd for $\mathrm{C}_{26} \mathrm{H}_{19} \mathrm{~N}_{3} \mathrm{O}_{2}$ : C, 77.02; H, 4.72; N, 10.36\%. Found: C, 77.04; $\mathrm{H}, 4.75$; N, $10.32 \%$.

9-Acetyl-11-amino-7-(4-methoxyphenyl)-8-methyl-7H-6b,9a(epoxyetheno)acenaphtho[1,2-b]pyrrole-10-carbonitrile 6 i. White powder, $\mathrm{mp}=252-254{ }^{\circ} \mathrm{C},{ }^{1} \mathrm{H} \mathrm{NMR}$ (DMSO- $d_{6}, 400 \mathrm{MHz}$ ) $\delta(\mathrm{ppm}): 2.22(\mathrm{~s}, 3 \mathrm{H}), 2.93(\mathrm{~s}, 3 \mathrm{H}), 3.74(\mathrm{~s}, 3 \mathrm{H}), 7.53(\mathrm{~d}, J=6.8 \mathrm{~Hz}$, $2 \mathrm{H}), 7.67(\mathrm{t}, J=7.6 \mathrm{~Hz}, 1 \mathrm{H}), 7.89(\mathrm{t}, J=7.4 \mathrm{~Hz}, 1 \mathrm{H}), 7.99-8.04(\mathrm{~m}$, $4 \mathrm{H}), 8.08(\mathrm{~s}, 2 \mathrm{H}), 8.338 .02(\mathrm{~d}, J=8.4 \mathrm{~Hz}, 2 \mathrm{H}) .{ }^{13} \mathrm{C}$ NMR (DMSO$\left.d_{6}, 100 \mathrm{MHz}\right) \delta(\mathrm{ppm}): 15.4,30.0,48.1,57.5,72.1,79.2,101.9$, $110.0,112.9,117.1,117.5,122.5,123.1,124.6,125.5,129.4$, $133.5,134.2$, 142.7, 152.5, 155.6, 158.8, 158.9, 166.1, 167.6, 177.6, 195.0. Anal. calcd for $\mathrm{C}_{27} \mathrm{H}_{21} \mathrm{~N}_{3} \mathrm{O}_{3}$ : C, 74.47; H, 4.86; N, 9.65\%. Found: C, 74.43; H, 4.89; N, 9.67\%.

Ethyl-11-amino-10-cyano-7-(2,6-dimethylphenyl)-8-methyl-7H6b,9a-(epoxyetheno)acenaphtho[1,2-b]pyrrole-9-carboxylate $\mathbf{6 j}$. White powder, $\mathrm{mp}=255-257{ }^{\circ} \mathrm{C},{ }^{1} \mathrm{H}$ NMR (DMSO- $d_{6}, 400 \mathrm{MHz}$ ) $\delta(\mathrm{ppm}): 1.35(\mathrm{t}, J=7.2 \mathrm{~Hz}, 3 \mathrm{H}), 2.21(\mathrm{~s}, 3 \mathrm{H}), 2.30(\mathrm{~s}, 3 \mathrm{H}), 2.33(\mathrm{~s}$, $3 \mathrm{H}), 3.31-3.34(\mathrm{~m}, 2 \mathrm{H}), 7.35(\mathrm{~d}, J=6.8 \mathrm{~Hz}, 2 \mathrm{H}), 7.55-7.59(\mathrm{~m}$, $2 \mathrm{H}), 7.77(\mathrm{t}, J=7.6 \mathrm{~Hz}, 2 \mathrm{H}), 7.84-7.89(\mathrm{~m}, 2 \mathrm{H}), 8.10(\mathrm{~s}, 2 \mathrm{H}), 8.17$ $(\mathrm{d}, J=7.6 \mathrm{~Hz}, 1 \mathrm{H}) .{ }^{13} \mathrm{C}$ NMR (DMSO- $\left.d_{6}, 100 \mathrm{MHz}\right) \delta(\mathrm{ppm}): 13.6$, 14.6, 21.4, 21.7, 59.6, 60.2, 72.0, 79.8, 104.3, 108.9, 112.8, 112.9, $116.8,117.0,121.5,123.7,124.1,125.3,125.6,128.4,133.9$, 
134.3, 135.2, 144.6, 152.3, 154.2, 167.6, 170.9, 179.4. Anal. calcd for $\mathrm{C}_{29} \mathrm{H}_{25} \mathrm{~N}_{3} \mathrm{O}_{3}$ : C, 75.14; $\mathrm{H}, 5.44 ; \mathrm{N}, 9.07 \%$. Found: $\mathrm{C}, 75.10 ; \mathrm{H}$, 5.42; N, 9.12\%.

Ethyl-11-amino-7-(4-chlorophenyl)-10-cyano-8-methyl-7 $\mathrm{H}$ 6b,9a-(epoxyetheno)acenaphtho[1,2-b]pyrrole-9-carboxylate 6k. White powder, $\mathrm{mp}=251-253{ }^{\circ} \mathrm{C},{ }^{1} \mathrm{H}$ NMR (DMSO- $d_{6}, 400$ MHz) $\delta(\mathrm{ppm}): 1.19(\mathrm{t}, J=7.2 \mathrm{~Hz}, 3 \mathrm{H}), 2.00(\mathrm{~s}, 3 \mathrm{H}), 4.04(\mathrm{q}, J=$ $7.2 \mathrm{~Hz}, 2 \mathrm{H}), 7.56(\mathrm{~d}, J=8.8 \mathrm{~Hz}, 1 \mathrm{H}), 7.65(\mathrm{~d}, J=7.2 \mathrm{~Hz}, 1 \mathrm{H}), 7.72$ $(\mathrm{t}, J=7.6 \mathrm{~Hz}, 1 \mathrm{H}), 7.77(\mathrm{~s}, 2 \mathrm{H}), 7.84(\mathrm{dd}, J=2.4 \mathrm{~Hz}, 8.8 \mathrm{~Hz}, 1 \mathrm{H})$, $7.90(\mathrm{t}, J=7.6 \mathrm{~Hz}, 1 \mathrm{H}), 8.03-8.06(\mathrm{~m}, 3 \mathrm{H}), 8.37$ (d, $J=8.0 \mathrm{~Hz}$, $2 \mathrm{H}) .{ }^{13} \mathrm{C}$ NMR (DMSO- $\left.d_{6}, 100 \mathrm{MHz}\right) \delta(\mathrm{ppm}): 13.7,14.6,59.8$, 60.2, 71.9, 79.2, 103.0, 108.9, 113.1, 116.9, 119.8, 123.5, 125.3, $126.1,134.1,136.2,142.4,151.2$, 152.4, 155.1, 158.7, 159.3, 167.2, 167.7, 180.2. Anal. calcd for $\mathrm{C}_{27} \mathrm{H}_{20} \mathrm{ClN}_{3} \mathrm{O}_{3}$ : C, 69.01; $\mathrm{H}$, 4.29; Cl, 7.54; N, 8.94\%. Found: C, 69.05; H, 4.26; N, 8.92\%.

Ethyl-11-amino-10-cyano-7-ethyl-8-methyl-7H-6b,9a-(epoxyetheno)acenaphtho[1,2-b]pyrrole-9-carboxylate 6l. White powder, $\mathrm{mp}=250-252{ }^{\circ} \mathrm{C},{ }^{1} \mathrm{H}$ NMR $\left(\mathrm{DMSO}-d_{6}, 400 \mathrm{MHz}\right)$ $\delta(\mathrm{ppm}): 0.76(\mathrm{t}, J=7.2 \mathrm{~Hz}, 3 \mathrm{H}), 1.44(\mathrm{t}, J=7.0 \mathrm{~Hz}, 3 \mathrm{H}), 2.25(\mathrm{~s}$, $3 \mathrm{H}), 2.81$ (q, $J=7.0 \mathrm{~Hz}, 2 \mathrm{H}), 4.30(\mathrm{q}, J=7.0 \mathrm{~Hz}, 2 \mathrm{H}), 7.57(\mathrm{~s}, 2 \mathrm{H})$, 7.66-7.71 (m, 1H), 7.72-7.76 (m, 2H), $7.79(\mathrm{t}, J=6.0 \mathrm{~Hz}, 1 \mathrm{H})$, $7.88(\mathrm{~d}, J=8.4 \mathrm{~Hz}, 1 \mathrm{H}), 8.03(\mathrm{~d}, J=8.0 \mathrm{~Hz}, 1 \mathrm{H}) .{ }^{13} \mathrm{C}$ NMR (DMSO- $\left.d_{6}, 100 \mathrm{MHz}\right) \delta$ (ppm): 7.9, 14.4, 14.6, 58.1, 58.6, 60.5, 70.7, 79.9, 110.4, 118.4, 120.6, 121.0, 124.7, 125.8, 127.8, 129.1, 129.6, 132.0, 135.8, 136.3, 142.7, 167.3, 168.1. Anal. calcd for $\mathrm{C}_{23} \mathrm{H}_{21} \mathrm{~N}_{3} \mathrm{O}_{3}$ : C, 71.30; H, 5.46; N, 10.85\%. Found: C, 71.28; H, 5.44; N, 10.88\%. IR (KBr) $\left(\nu_{\max }, \mathrm{cm}^{-1}\right): 3405$ and $3278\left(\mathrm{NH}_{2}\right)$, $2197(\mathrm{CN}), 1663(\mathrm{C}=\mathrm{O}), 1639(\mathrm{OC}=\mathrm{C})$.

9-Acetyl-11-amino-7-ethyl-8-methyl-7H-6b,9a-(epoxyetheno)acenaphtho[1,2-b]pyrrole-10-carbonitrile $6 \mathrm{~m}$. White powder, $\mathrm{mp}=$ 251-253 ${ }^{\circ} \mathrm{C},{ }^{1} \mathrm{H}$ NMR (DMSO- $\left.d_{6}, 400 \mathrm{MHz}\right) \delta(\mathrm{ppm}): 0.86(\mathrm{t}, J=$ $7.0 \mathrm{~Hz}, 3 \mathrm{H}), 2.32(\mathrm{~s}, 3 \mathrm{H}), 2.36(\mathrm{~s}, 3 \mathrm{H}), 3.81(\mathrm{q}, J=7.1 \mathrm{~Hz}, 2 \mathrm{H})$, $7.46(\mathrm{~s}, 2 \mathrm{H}), 7.63(\mathrm{t}, J=7.6 \mathrm{~Hz}, 1 \mathrm{H}), 7.70(\mathrm{~d}, J=7.2 \mathrm{~Hz}, 1 \mathrm{H}), 7.75$ (t, $J=7.0 \mathrm{~Hz}, 1 \mathrm{H}), 7.85(\mathrm{~d}, J=7.6 \mathrm{~Hz}, 2 \mathrm{H}), 8.01(\mathrm{~d}, J=7.6 \mathrm{~Hz}$, $1 \mathrm{H}) .{ }^{13} \mathrm{C}$ NMR (DMSO- $\left.d_{6}, 100 \mathrm{MHz}\right) \delta$ (ppm): 15.3, 15.9, 30.3, 58.4, 59.0, 72.2, 79.9, 118.5, 120.4, 121.4, 121.6, 124.4, 124.9, 127.7, 128.9, 129.5, 131.9, 135.9, 136.3, 143.0, 167.2, 192.7. Anal. calcd for $\mathrm{C}_{22} \mathrm{H}_{19} \mathrm{~N}_{3} \mathrm{O}_{2}$ : C, 73.93; $\mathrm{H}, 5.36 ; \mathrm{N}, 11.76 \%$. Found: $\mathrm{C}$, 73.92; H, 5.39; N, 11.73\%.

Ethyl-11-amino-7-butyl-10-cyano-8-methyl-7H-6b,9a-(epoxyetheno)acenaphtho[1,2-b]pyrrole-9-carboxylate 6n. White powder, $\mathrm{mp}=247-249{ }^{\circ} \mathrm{C},{ }^{1} \mathrm{H}$ NMR $\left(\mathrm{DMSO}-d_{6}, 400 \mathrm{MHz}\right)$ $\delta$ (ppm): 0.95 (t, $J=7.2 \mathrm{~Hz}, 3 \mathrm{H}), 1.44(\mathrm{t}, J=7.2 \mathrm{~Hz}, 3 \mathrm{H}), 1.80-$ $1.92(\mathrm{~m}, 4 \mathrm{H}), 2.25(\mathrm{~s}, 3 \mathrm{H}), 2.98(\mathrm{t}, J=6.4 \mathrm{~Hz}, 2 \mathrm{H}), 4.32(\mathrm{q}, J=$ $7.2 \mathrm{~Hz}, 2 \mathrm{H}), 7.57(\mathrm{~s}, 2 \mathrm{H}), 7.67(\mathrm{t}, J=7.6 \mathrm{~Hz}, 1 \mathrm{H}), 7.71(\mathrm{~d}, J=$ $7.6 \mathrm{~Hz}, 1 \mathrm{H}), 7.75(\mathrm{~d}, J=6.4 \mathrm{~Hz}, 1 \mathrm{H}), 7.79(\mathrm{t}, J=6.4 \mathrm{~Hz}, 1 \mathrm{H}), 7.88$ $(\mathrm{d}, J=8.0 \mathrm{~Hz}, 1 \mathrm{H}), 8.03(\mathrm{~d}, J=8.0 \mathrm{~Hz}, 1 \mathrm{H}) .{ }^{13} \mathrm{C}$ NMR $\left(\mathrm{DMSO}_{6} d_{6}\right.$, $100 \mathrm{MHz}) \delta$ (ppm): 12.7, 14.4, 14.6, 19.0, 26.2, 55.1, 58.1, 60.5, 70.7, 79.9, 110.4, 118.4, 120.6, 121.0, 124.7, 125.8, 127.8, 129.1, 129.6, 132.0, 135.8, 136.3, 142.7, 167.3, 168.1. Anal. calcd for $\mathrm{C}_{25} \mathrm{H}_{25} \mathrm{~N}_{3} \mathrm{O}_{3}$ : C, 72.27; H, 6.06; N, 10.11\%. Found: C, 72.30; H, 6.05; N, 10.08\%. IR (KBr) $\left(\nu_{\max }, \mathrm{cm}^{-1}\right): 3389$ and $3179\left(\mathrm{NH}_{2}\right)$, $2203(\mathrm{CN}), 1661(\mathrm{C}=\mathrm{O}), 1635(\mathrm{OC}=\mathrm{C})$.

9-Acetyl-11-amino-7-butyl-8-methyl-7H-6b,9a-(epoxyetheno) acenaphtho[1,2-b]pyrrole-10-carbonitrile 6o. White powder, mp $=248-250{ }^{\circ} \mathrm{C},{ }^{1} \mathrm{H}$ NMR (DMSO- $\left.d_{6}, 400 \mathrm{MHz}\right) \delta(\mathrm{ppm}): 0.84(\mathrm{t}, J=$ $7.2 \mathrm{~Hz}, 3 \mathrm{H}), 1.05-1.12(\mathrm{~m}, 4 \mathrm{H}), 2.31(\mathrm{~s}, 3 \mathrm{H}), 2.36$ (s, 3H), 2.96 (q, $J$
$=7.3 \mathrm{~Hz}, 2 \mathrm{H}), 7.46(\mathrm{~s}, 2 \mathrm{H}), 7.63(\mathrm{t}, J=7.6 \mathrm{~Hz}, 1 \mathrm{H}), 7.70(\mathrm{~d}, J=$ $7.2 \mathrm{~Hz}, 1 \mathrm{H}), 7.75$ (t, $J=7.0 \mathrm{~Hz}, 1 \mathrm{H}), 7.85(\mathrm{~d}, J=7.2 \mathrm{~Hz}, 2 \mathrm{H}), 8.01$ $(\mathrm{d}, J=8.0 \mathrm{~Hz}, 1 \mathrm{H}) .{ }^{13} \mathrm{C}$ NMR (DMSO- $\left.d_{6}, 100 \mathrm{MHz}\right) \delta(\mathrm{ppm}): 15.2$, 15.9, 23.7, 29.9, 30.3, 58.4, 59.5, 72.2, 79.9, 118.5, 120.4, 121.4, 121.6, 124.4, 124.9, 127.7, 128.9, 129.5, 131.9, 135.9, 136.3, 166.6, 167.2, 192.7. Anal. calcd for $\mathrm{C}_{24} \mathrm{H}_{23} \mathrm{~N}_{3} \mathrm{O}_{2}$ : C, 74.78; $\mathrm{H}$, 6.01 ; N, 10.90\%. Found: C, 74.75; H, 6.03; N, 10.92\%.

Ethyl-9-acetyl-11-amino-7-benzyl-8-methyl-7H-6b,9a-(epoxyetheno)acenaphtho[1,2-b]pyrrole-10-carboxylate $6 \mathbf{p}$. White powder, $\mathrm{mp}=249-251{ }^{\circ} \mathrm{C},{ }^{1} \mathrm{H}$ NMR $\left(\mathrm{DMSO}-d_{6}, 400 \mathrm{MHz}\right)$ $\delta(\mathrm{ppm}): 0.83(\mathrm{t}, J=7.0 \mathrm{~Hz}, 3 \mathrm{H}), 2.10(\mathrm{~s}, 3 \mathrm{H}), 2.44(\mathrm{~s}, 3 \mathrm{H}), 3.80(\mathrm{q}$, $J=7.3 \mathrm{~Hz}, 2 \mathrm{H}), 4.84(\mathrm{~d}, J=16.0 \mathrm{~Hz}, 1 \mathrm{H}), 5.12(\mathrm{~d}, J=16.0 \mathrm{~Hz}$, $1 \mathrm{H}), 7.42(\mathrm{~s}, 2 \mathrm{H}), 7.64-7.67(\mathrm{~m}, 2 \mathrm{H}), 7.70-7.74(\mathrm{~m}, 3 \mathrm{H}), 7.84(\mathrm{~d}, J$ $=6.4 \mathrm{~Hz}, 3 \mathrm{H}), 7.99-8.06(\mathrm{~m}, 3 \mathrm{H}) .{ }^{13} \mathrm{C} \mathrm{NMR}$ (DMSO- $\left.d_{6}, 100 \mathrm{MHz}\right)$ $\delta$ (ppm): 15.0, 15.1, 31.9, 58.7, 59.4, 71.8, 72.5, 80.0, 118.2, 118.4, $120.1,120.8$, 124.0, 124.1, 125.6, 127.5, 128.6, 129.1, 131.8, 136.2, 136.6, 142.9, 163.4, 166.1, 166.7, 167.3, 167.9, 195.3. Anal. calcd for $\mathrm{C}_{29} \mathrm{H}_{26} \mathrm{~N}_{2} \mathrm{O}_{4}$ : C, 74.66; H, 5.62; N, 6.00\%. Found: C, 74.68; H, 5.59; N, 6.02\%. IR (KBr) $\left(\nu_{\max }, \mathrm{cm}^{-1}\right): 3359$ and 3161 $\left(\mathrm{NH}_{2}\right), 1738(\mathrm{C}=\mathrm{O}), 1667(\mathrm{C}=\mathrm{O}), 1639(\mathrm{OC}=\mathrm{C})$.

9-Ethyl-10-methyl-11-amino-7-ethyl-8-methyl-7H-6b,9a-(epoxyetheno)acenaphtho[1,2-b]pyrrole-9,10-dicarboxylate 6q. White powder, $\mathrm{mp}=247-249{ }^{\circ} \mathrm{C},{ }^{1} \mathrm{H}$ NMR $\left(\mathrm{DMSO}-d_{6}, 400 \mathrm{MHz}\right)$ $\delta(\mathrm{ppm}): 0.86(\mathrm{t}, J=7.0 \mathrm{~Hz}, 3 \mathrm{H}), 1.18(\mathrm{t}, J=7.2 \mathrm{~Hz}, 3 \mathrm{H}), 2.00(\mathrm{~s}$, $3 \mathrm{H}), 3.22(\mathrm{~s}, 3 \mathrm{H}), 3.81(\mathrm{q}, J=7.1 \mathrm{~Hz}, 2 \mathrm{H}), 4.04(\mathrm{q}, J=7.2 \mathrm{~Hz}, 2 \mathrm{H})$, $7.38(\mathrm{~s}, 2 \mathrm{H}), 7.59(\mathrm{t}, J=7.6 \mathrm{~Hz}, 1 \mathrm{H}), 7.63(\mathrm{~d}, J=7.2 \mathrm{~Hz}, 1 \mathrm{H}), 7.69$ (t, $J=6.6 \mathrm{~Hz}, 1 \mathrm{H}), 7.81(\mathrm{~d}, J=8.4 \mathrm{~Hz}, 1 \mathrm{H}), 7.99$ (d, $J=7.6 \mathrm{~Hz}$, $1 \mathrm{H}), 8.08(\mathrm{~d}, J=6.8 \mathrm{~Hz}, 1 \mathrm{H}) .{ }^{13} \mathrm{C}$ NMR (DMSO- $d_{6}, 100 \mathrm{MHz}$ ) $\delta$ (ppm): 14.5, 14.6, 15.1, 58.6, 60.1, 60.9, 61.7, 70.8, 79.8, 120.1, 124.0, 124.2, 126.0, 127.6, 128.5, 129.0, 131.7, 136.1, 136.6, 142.7, 164.6, 165.0, 166.2, 166.9. Anal. calcd for $\mathrm{C}_{24} \mathrm{H}_{24} \mathrm{~N}_{2} \mathrm{O}_{5}: \mathrm{C}$, 68.56; H, 5.75; N, 6.66\%. Found: C, 68.58; H, 5.77; N, 6.63\%. IR $(\mathrm{KBr})\left(\nu_{\max }, \mathrm{cm}^{-1}\right): 3412$ and $3249\left(\mathrm{NH}_{2}\right), 1736(\mathrm{C}=\mathrm{O}), 1675(\mathrm{C}=$ $\mathrm{O}), 1642(\mathrm{OC}=\mathrm{C})$.

\section{Conclusions}

In conclusion, we have reported a highly efficient method for the synthesis of biologically important propellane derivatives via a one-pot, sequential four-component condensation reaction presence of triethylamine in ethanol at room temperature. High atom-economy, simple procedure in the excellent yields, easy workup procedure, mild reaction conditions and metalcatalyst free are the main advantages of this method.

\section{Conflicts of interest}

There are no conflicts to declare.

\section{Acknowledgements}

The authors thank Persian Gulf University Research Councils for the financial support of this work.

\section{References}

1 D. B. Ramachary and M. Kishor, J. Org. Chem., 2007, 72, 5056. 
2 (a) L. D. S. Yadav, S. Singh and V. K. Rai, Green Chem., 2009, 11, 878; (b) W. Leitner, Green Chem., 2009, 11, 603.

3 A. Hasaninejad, A. Zare and M. Shekouhy, Tetrahedron, 2011, 67, 390.

4 B. Maleki, G. Esmailian and R. Tayebee, Org. Prep. Proced. Int., 2015, 47, 461.

5 M. Dabiri, Z. N. Tisseh, M. Bahramnejad and A. Bazgir, Ultrason. Sonochem., 2011, 18, 1153.

$6 \mathrm{~J}$. Zhu and H. Bienayme, Multicomponent Reactions, John Wiley \& Sons, 2006.

7 T. Amanpour, A. Bazgir, A. M. Ardekani and R. Ghahremanzadeh, Monatsh. Chem., 2014, 145, 627.

8 V. A. Chebanov, E. A. Muravyova, S. M. Desenko, V. I. Musatov, I. V. Knyazeva, S. V. Shishkina, O. V. Shishkin and C. O. Kappe, J. Comb. Chem., 2006, 8, 427. 9 J. M. Jamison, K. Krabill, A. Hatwalkar, E. Jamison and C. Tsai, Cell Biol. Int. Rep., 1990, 14, 1075.

10 S. M. Menchen, S. C. Benson, J. Y. L. Lam, W. Zhen, D. Sun, B. B. Rosenblum, S. H. Khan and M. Taing, US Pat., US 6583168, 2003transChem. Abstr. 2003, 139, 54287f.

11 G. W. Rewcastle, G. J. Atwell, L. Zhuang, B. C. Baguley and W. A. Denny, J. Med. Chem., 1991, 34, 217.

12 R. J. Sarma and J. B. Baruah, Dyes Pigm., 2005, 64, 91. 13 J. B. Laursen and J. Nielsen, Chem. Rev., 2004, 104, 1663.

14 C. Neves-Pinto, V. Malta, M. Pinto, R. Santos, S. Castro and A. Pinto, J. Med. Chem., 2002, 45, 740.

15 D. Cartwright, W. Chilton and D. Benson, Appl. Microbiol. Biotechnol., 1995, 43, 211.

16 M. Muller and T. Sorrell, Prostaglandins, 1995, 50, 301. 17 G. A. Kraus and I. A. Kim, J. Org. Chem., 2003, 68, 4517.

18 N. Vicker, L. Burgess, I. S. Chuckowree, R. Dodd, A. J. Folkes, D. J. Hardick, T. C. Hancox, W. Dangerfield, C. Liddle, P. Mistry, A. J. Stewart and W. A. Denny, J. Med. Chem., 2002, 45, 721.

19 Y. F. Qiao, T. Okazaki, T. Ando, K. Mizoue, K. Kondo, T. Eguchi and K. Kakinuma, J. Antibiot., 1998, 51, 282.

20 J. P. Poupelin, G. S. Ruf, O. F. Blanpin, G. Narcisse, G. U. Ernouf and R. Lacroix, Eur. J. Med. Chem., 1978, 13, 67.

21 (a) J. Altman, E. Babad, J. Itzchaki and D. Ginsburg, Tetrahedron, 1966, 279; (b) R. W. Weber and J. M. Cook, Can. J. Chem., 1977, 56, 189; (c) K. B. Wiberg, J. Am. Chem. Soc., 1983, 105, 1227; (d) A. I. Ioffe, V. A. Svyatkin and O. M. Nefedov, Russ. Chem. Bull., 1988, 37, 1827; (e) M. Shin, M. H. Kim, T. Ha, J. Jeon, K. H. J. S. Chung Kim and Y. G. Kim, Tetrahedron, 2014, 70, 1617; $(f)$ D. Kinsberg, Acc. Chem. Res., 1974, 7, 286; (g) K. B. Wiberg, Chem. Rev., 1989, 89, 975; (h) J. P. Ainoliisa and M. P. K. Ari, Tetrahedron, 2005, 61, 8769.

22 (a) T. Ibuka, K. Tanaka and Y. Inubushi, Chem. Pharm. Bull., 1974, 22, 782; (b) H.-Y. Lee, D.-I. Kim and S. Kim, J. Chem. Soc., Chem. Commun., 1996, 1539; (c) L. Zu, B. W. Boal and N. K. Garg, J. Am. Chem. Soc., 2011, 133, 8877; (d) H. Jian
Mei, Y. Ritsuko, Y. Chun-shu and Y. Fukuyama, Tetrahedron Lett., 2000, 41, 6111.

23 A. Rezvanian and A. Alizadeh, Tetrahedron, 2012, 68, 10164. 24 (a) K. Weniges, P. Guenther, W. Kasel, G. Hubertus and P. Gunther, Angew. Chem., Int. Ed. Engl., 1981, 20, 960; (b) J.-M. Huang, C.-S. Yang, M. Tanaka and Y. Fukuyama, Tetrahedron, 2001, 57, 4691; (c) K. C. Nicolaou, S. A. Snyder, T. Montagnon and G. Vassilikogiannakis, Angew. Chem., Int. Ed., 2002, 41, 1668; (d) B. M. Trost and Y. Shi, J. Am. Chem. Soc., 1991, 113, 701; (e) K. Asahi and H. Nishino, Tetrahedron, 2008, 64, 1620.

25 A. Alizadeh, A. Rezvanian and L. G. Zhu, J. Org. Chem., 2012, $77,4385$.

26 A. Rezvanian and A. Alizadeh, Tetrahedron, 2012, 68, 10164. 27 A. Alizadeh, F. Bayat, L. Moafi and L. G. Zhu, Tetrahedron, 2015, 71, 8150.

28 I. Yavari and A. Khajeh-Khezri, Mol. Diversity, 2017, 21, 849. 29 A. A. Hassan, N. K. Mohamed, L. E. Abd El-Haleem, S. Brase and M. Nieger, Curr. Org. Synth., 2016, 13, 426.

30 M. Sirouspour and S. Souri, J. Heterocycl. Chem., 2016, 53, 147.

31 L. J. Zhang and C. G. Yan, Tetrahedron, 2013, 69, 4915.

32 I. Yavari, A. Khajeh-Khezri, S. Bahemmat and M. R. Halvagar, Synlett, 2017, 28, 1785.

33 I. Yavari, A. Khajeh-Khezri and M. R. Halvagar, Arabian J. Chem., 2018, 11, 188.

34 J. Wang, H. Liu, R. Wen, J. Li and S. Zhu, Chin. J. Chem., 2017, 35, 1463.

35 E. Safari, A. Maryamabadi and A. Hasaninejad, RSC Adv., 2017, 7, 39502.

36 M. Beyrati and A. Hasaninejad, Tetrahedron Lett., 2017, 58, 1947.

37 A. Maryamabadi, A. Hasaninejad, N. Nowrouzi and G. Mohebbi, Bioorg. Med. Chem., 2017, 25, 2057.

38 M. Beyrati, M. Forutan, A. Hasaninejad, E. Rakovský, S. Babaei, A. Maryamabadi and G. Mohebbi, Tetrahedron, 2017, 73, 5144.

39 A. Hasaninejad and M. Beyrati, RSC Adv., 2018, 8, 1934.

40 A. Maryamabadi, A. Hasaninejad, N. Nowrouzi, G. Mohebbi and B. Asghari, Bioorg. Med. Chem., 2016, 24, 1408.

41 M. Beyrati and A. Hasaninejad, Org. Prep. Proced. Int., 2016, 48, 393.

42 A. Hasaninejad, N. Golzar, M. Beyrati, A. Zare and M. M. Doroodmand, J. Mol. Catal. A: Chem., 2013, 372, 137. 43 A. Hasaninejad and S. Firoozi, Mol. Diversity, 2013, 17, 499. 44 A. Hasaninejad, A. Zare, M. Shekouhy and J. Ameri Rad, Green Chem., 2011, 13, 958.

45 S. Mojikhalifeh and A. Hasaninejad, Tetrahedron Lett., 2017, 58, 2574.

46 S. Mojikhalifeh and A. Hasaninejad, Org. Chem. Front., 2018, DOI: 10.1039/C8QO00210J.

47 A. Hasaninejad, S. Mojikhalifeh and M. Beyrati, Appl. Organomet. Chem., 2018, DOI: 10.1002/aoc.4380. 